This is a Post-Print Copy of:

Wouter van der Wijngaart, Dipankar Chugh, Elisabeth Man, Jessica Melin, and Göran Stemme, "A Low-Temperature Thermopneumatic Actuation Principle for Gas Bubble Microvalves", JOURNAL OF MICROELECTROMECHANICAL SYSTEMS, VOL. 16, NO. 3, JUNE 2007

Doi: 10.1109/JMEMS.2007.893514

http://ieeexplore.ieee.org/xpls/abs_all.jsp?arnumber=4276821\&tag=1

(c) 2007 IEEE. Personal use of this material is permitted. Permission from IEEE must be obtained for all other users, including reprinting/ republishing this material for advertising or promotional purposes, creating new collective works for resale or redistribution to servers or lists, or reuse of any copyrighted components of this work in other works. 


\title{
A Low-Temperature Thermopneumatic Actuation Principle for Gas Bubble Microvalves
}

\author{
Wouter van der Wijngaart, Member, IEEE, Dipankar Chugh, Elisabeth Man, \\ Jessica Melin, and Göran Stemme, Fellow, IEEE
}

\begin{abstract}
We introduce a novel type of microfluidic actuator in which a trapped air bubble functions as the thermally controlled volume displacing element. The pressure of the trapped air pocket is controlled by the changing equilibrium gas composition for static operation and by thermal expansion for dynamic operation. The volume displacement is determined by the liquid surface tension and the valve geometry. A fully functional demonstrator device was successfully fabricated and tested. The absence of moving mechanical parts, the electrical control of the valve, and the limited required actuation temperature make the actuator suitable for control of large-scale integration fluidic networking in biotechnical applications.

[2006-0090]
\end{abstract}

Index Terms-Bubble valve, microfluidics, microvalve, surface tension, thermopneumatic.

\section{INTRODUCTION}

$\mathbf{M}$ ICROVALVES form one of the fundamental components of microfluidic-based devices. Active liquid microvalves are used not only to control flow in microchannels, but also to perform peristaltic pumping [1] or seal off reaction zones [2].

Gas bubbles have been used as active elements in reversible volume-displacing microfluidic actuators. The bubbles are commonly formed and controlled by a liquid-gas phase change either through boiling or electrolysis [3]. Handique et al. [4] introduced a new type of gas bubble actuator for pumping of discrete drops, in which the active gas bubble consists of air that is trapped during device priming. For micropump applications, the trapped air pocket volume must be large enough to provide the minimum required pump stroke. Moreover, a cyclic pumping scheme limits the time for the trapped gas and the pumped liquid to reach phase equilibrium; thus, the actuation relies on pure thermal expansion of the gas, whereas the contribution of liquid-gas mass transport to the pump pressure is negligible.

In this paper, we introduce thermopneumatic actuation of trapped air for valving of on-chip liquids. This approach leads to a number of obvious benefits. The volume-displacing bubble actuator utilizes both pure thermal expansion and vapor pressure saturation, allowing device control at much lower temperatures than previously reported [4]. Because no pump stroke must be generated, the trapped air pocket can be small, and the actuation scheme allows downscaling microvalve elements to a size of $10 \mu \mathrm{m}$ or below. The valves are controlled electrically, thus not requiring costly or complex (off-chip) pneumatic interfacing and control equipment [1], [2]. The novel approach is thus suited for large-scale integration (LSI) of active fluidic control elements. In comparison to previous microvalves, low power is required because no boiling heat is required and no inherent stiffness of mechanical diaphragm or membrane must be accounted for. Neither does the method require integrated catalyst material for reversed hydrolysis [5].

The moderate temperatures required to operate the valves make them suitable for biological assays in particular, where samples are prone to thermal denaturation. The actuation principle here is independent from actuation principles that require electrical fields or field gradients in the liquid, such as electroosmotically controlled flow, thus making the combination of such actuation schemes uncomplicated.

\section{Device Description ANd Operation Principle}

Fig. 1 shows a schematic of a microvalve using the novel actuator. It consists of a liquid microchannel with an inlet and an outlet. An actuation window connects the liquid channel with an actuation chamber. The resistive heater in the actuation chamber is used for thermopneumatic actuation.

During liquid priming of the channel, a combination of surface wettability and geometry control ensures that the liquid-air-solid triphase line remains in the actuation window between the liquid channel and the actuation chamber, i.e., the liquid will not enter the actuation chamber, thus trapping an air pocket in the actuation chamber. The valve is open in the normal mode of operation. Closing the valve is achieved by increasing the temperature of the gas volume in the actuation chamber, which increases the pressure of the gas. The pressure increase introduces a gas bubble into the liquid channel, blocking the flow. The actuation mechanism is similar to that of a thermopneumatic diaphragm valve [6], except that in our device, there is no mechanical part such as a membrane or a diaphragm. The liquid-air interface at the actuation window itself acts as a mechanical barrier between the actuation chamber and the liquid channel. Liquid flow in the channel is again established when the gas pressure is reduced by decreasing the temperature in the actuation chamber. The microchannel downstream of the 


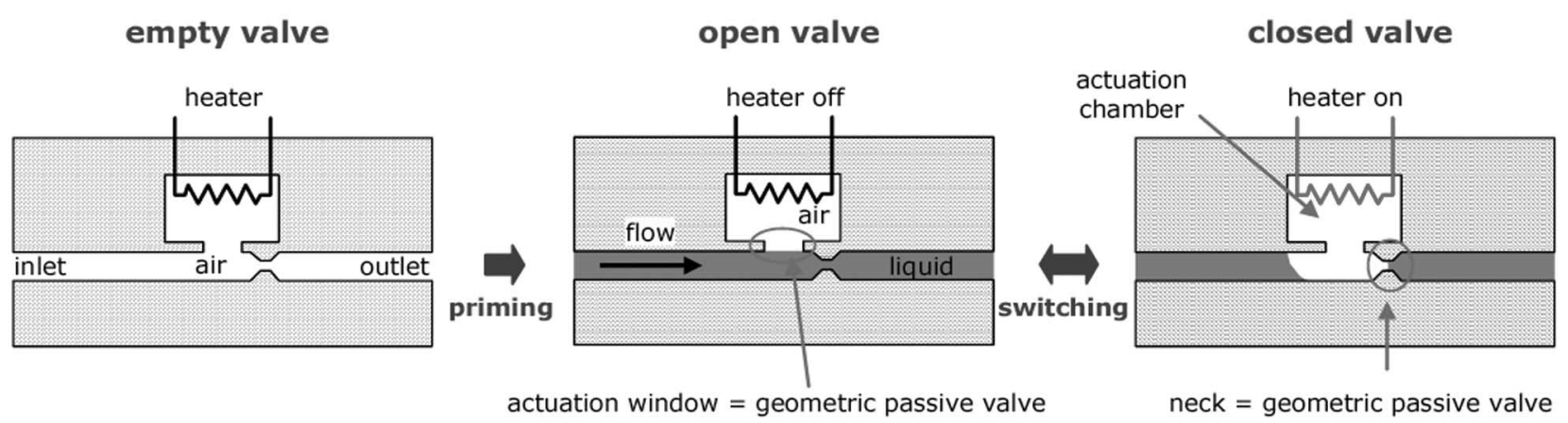

Fig. 1. Conceptual schematic of the (left) microvalve device, same device after priming, (middle) valve being unactuated in the open state, and (right) device being in the actuated closed state.

actuation window is necked such that large capillary forces hold the bubble at the actuation window and prevent it from entering the downstream system [7].

\section{Theoretical Device Performance}

A theoretical analysis of the device was performed to understand the influence of the gas temperature $T$ on the actuation chamber pressure $P_{g}$ and the volume displacement $\Delta V$. We assume a two-phase water-air system consisting of a finite closed gas volume

$$
V=V_{0}+\Delta V
$$

in the actuation chamber and a large (infinite) amount of liquid in the channel (Fig. 2). Table I overviews the variables and constants that are used throughout this paper.

As long as the temperature is between the freezing point and the boiling point of water, the system is a water vapor two-phase system. The enclosed gas consists of a mixture of air and water vapor. The liquid in the channel consists of water with dissolved air constituents. In the following analysis, we will start by assuming that the system is in thermodynamic stationary state and that all state changes are quasi-static. The model approach we use determines the thermodynamic equilibrium state of each of the vapor and air constituents independently from one another. The gas pressure $P_{g}$ in the actuation chamber can be expressed as

$$
P_{g}=\sum_{i} P_{i}+P_{v}=P_{a}+P_{v} .
$$

When increasing the actuation chamber temperature, water will evaporate at the water-air interface, and the vapor pressure increases until it reaches the saturation pressure. At the same time, air constituents will diffuse through the water until stationary state is reached.

We will further assume that the thermodynamic properties are equal in every point of the actuation chamber. We will also assume that the temperature in a liquid layer close to the gas interface is approximately at the gas temperature $T_{g}$ and that the temperature profile in the liquid is stationary (the exact shape of the temperature profile is not of interest in this model).

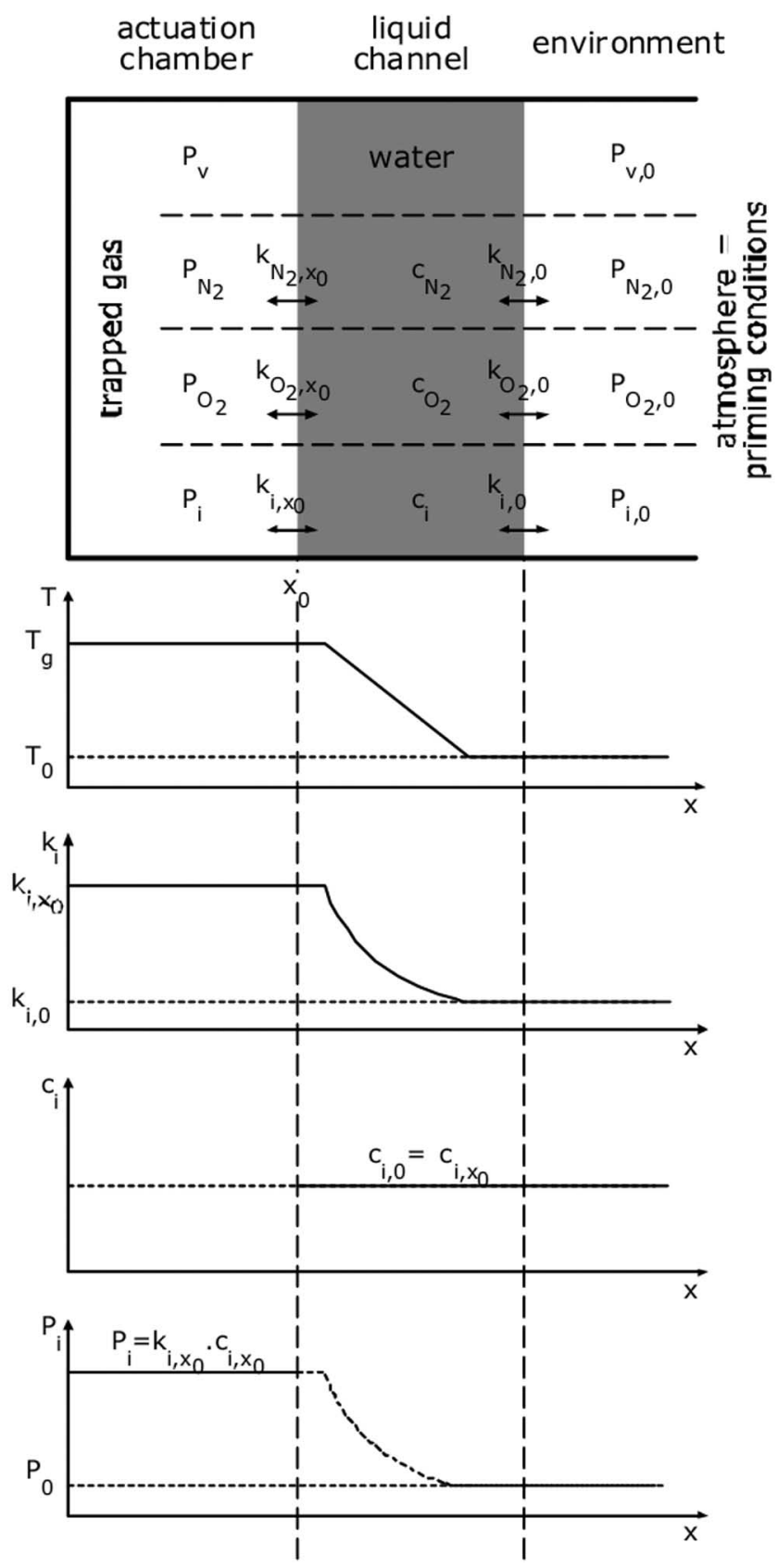

Fig. 2. Schematic of the two-phase model. The values of $T_{0}, T_{g}, k_{i, 0}$, and $c_{i, 0}$ are defined by the heater temperature and the atmospheric conditions at priming. 
TABLE I

OVERVIEW OF VARIABLES AND CONSTANTS

\begin{tabular}{|c|l|}
\hline 0 & Index indicating initial conditions, e.g. room temperature or atmospheric pressure. \\
\hline$i$ & Index indicating a specific constituting gas of dry air, i.e. $\mathrm{N}_{2}, \mathrm{O}_{2}, \mathrm{CO}_{2}, \mathrm{Ar} .$. \\
\hline$a$ & Index indicating dry air in its normal composition \\
\hline$v$ & Index indicating water vapour \\
\hline$g$ & Index indicating the total gas mixture \\
\hline$l$ & Index indicating the liquid phase \\
\hline$w$ & $\begin{array}{l}\text { Characteristic size of the device actuation window. } w \text { is the actuation window diameter for } \\
\text { a circular actuation window shape; } w \text { is the smallest rectangle side for a rectangular } \\
\text { actuation window shape. }\end{array}$ \\
\hline$h$ & The height of the liquid channel. \\
\hline$V$ & The total gas volume of the actuation chamber \\
\hline$V_{0}$ & The initial gas volume of the actuation chamber (non-actuated) \\
\hline$\Delta V$ & The gas volume change $V$ - $V_{0}$ at the actuation window during actuation \\
\hline$P_{(i, v)}$ & The (partial) gas pressure (of constituent $i$ or vapour) in the actuation chamber \\
\hline$n$ & Molar fraction \\
\hline$R$ & The ideal gas constant $=8.3145 \mathrm{~J} \cdot \mathrm{mol}^{-1} \cdot \mathrm{K}^{-1}$ \\
\hline$T$ & The absolute gas temperature. \\
\hline$T_{c l o s e}$ & The gas temperature for which a valve closes. \\
\hline$\Delta H_{i, s o l}$ & The enthalpy of solution for gas constituent $i$ in water. \\
\hline$\Delta H_{v a p}$ & The liquid-gas phase change enthalpy for water. \\
\hline$c_{i}$ & The concentration of gas $i$ in water \\
\hline$k_{i}$ & Henry's law constant of the gas i. \\
\hline$\gamma$ & Liquid surface tension. $\gamma \approx 0.025 \mathrm{Nm}^{-1}$ for the liquid used during device testing. \\
\hline$r_{l}, r_{2}$ & Radii of two perpendicular planes in the liquid surface. \\
\hline$r$ & Radius of a spherical liquid-air interface $\left(r=r_{l}=r_{2}\right)$. \\
\hline$r_{c h}$ & Radius of a cylindrical liquid channel. \\
\hline
\end{tabular}

The saturation vapor pressure $P_{v}$ can be expressed by the volume-independent Clausius-Clapeyron equation

$$
P_{v}=P_{v, 0} \cdot \exp \left[\frac{\Delta H_{\mathrm{vap}}}{R}\left(\frac{1}{T_{0}}-\frac{1}{T_{g}}\right)\right] .
$$

The partial pressures $P_{i}$ depend on the solubility of the constituent in water, according to (temperature dependent) Henry's law [8], i.e.,

$$
P_{i}=k_{i}(T) \cdot c_{i}=k_{i, 0} \cdot \exp \left[\frac{\Delta H_{i, \mathrm{sol}}}{R}\left(\frac{1}{T_{0}}-\frac{1}{T_{g}}\right)\right] \cdot c_{i} .
$$

We can now assume that in stationary state, the concentration of the dissolved gasses throughout the liquid is constant and in equilibrium with the environment, i.e.,

$$
c_{i}=c_{i, 0}=\frac{P_{i, 0}}{k_{i, 0}} .
$$

Equation (4) can thus be rewritten as

$$
P_{i}=P_{i, 0} \cdot \exp \left[\frac{\Delta H_{i, \mathrm{sol}}}{R}\left(\frac{1}{T_{0}}-\frac{1}{T_{g}}\right)\right] .
$$

Combining (2), (3), and (6), the pressure-temperature relation of the actuation chamber can now be written as

$$
P_{g, \text { stat }}=\sum_{j=i, v} P_{j, 0} \cdot \exp \left[\frac{\Delta H_{j}}{R}\left(\frac{1}{T_{0}}-\frac{1}{T}\right)\right]
$$

in which $\Delta H_{j}$ is the phase change enthalpy $\left(\Delta H_{j}=\Delta H_{\text {vap }}\right.$ for water, and $\Delta H_{j}=\Delta H_{i, \text { sol }}$ for the air constituents).

Equation (7) describes the gas pressure in the stationary state of the system. The speed of reaching this stationary state for the water component is proportional to the geometrical size of the actuation window and inversely proportional to the actuation chamber volume. The speed of reaching the stationary state for the air constituents is inversely proportional to both the actuation chamber volume and the diffusion path length of the constituents through the liquid.

The behavior of gas components that are slow in reaching the stationary state can be described with the ideal gas law

$$
P_{j} V_{j}=n_{j} R T_{g}
$$

with $j=i$ or $v$ and in which $n_{j}$ is constant. For systems where $V_{0} \gg \Delta V$, the value for $P_{j}$ can be expressed with the Gay-Lussac law

$$
P_{j, \mathrm{dyn}}=P_{j, 0}+\frac{P_{j, 0}}{T_{j, 0}}\left(T_{g}-T_{0}\right) .
$$

We will call such system behavior "dynamic." Thus, the dynamic behavior is determined by the pure thermal expansion of the gas, as described by (9), whereas the static behavior is determined by the saturation vapor pressure, as described by (7).

The change from dynamic to static behavior complicates the control of the valve, and a system where the dynamic behavior of the gas constituents is either very short or very long as compared to the valve closing time of interest would be preferable.

In Fig. 3, the theoretical absolute and relative gas pressures in the actuation chamber are plotted versus the temperature according to (6), (7), and (9). The analysis shows that a stationary pressure above $1 \mathrm{~atm}$ can be generated with a temperature rise of only $42{ }^{\circ} \mathrm{C}$. The figure also illustrates the large difference in pressure range between dynamic and stationary operations of the valve; care must be taken for which of these two regimes the valve is designed. 


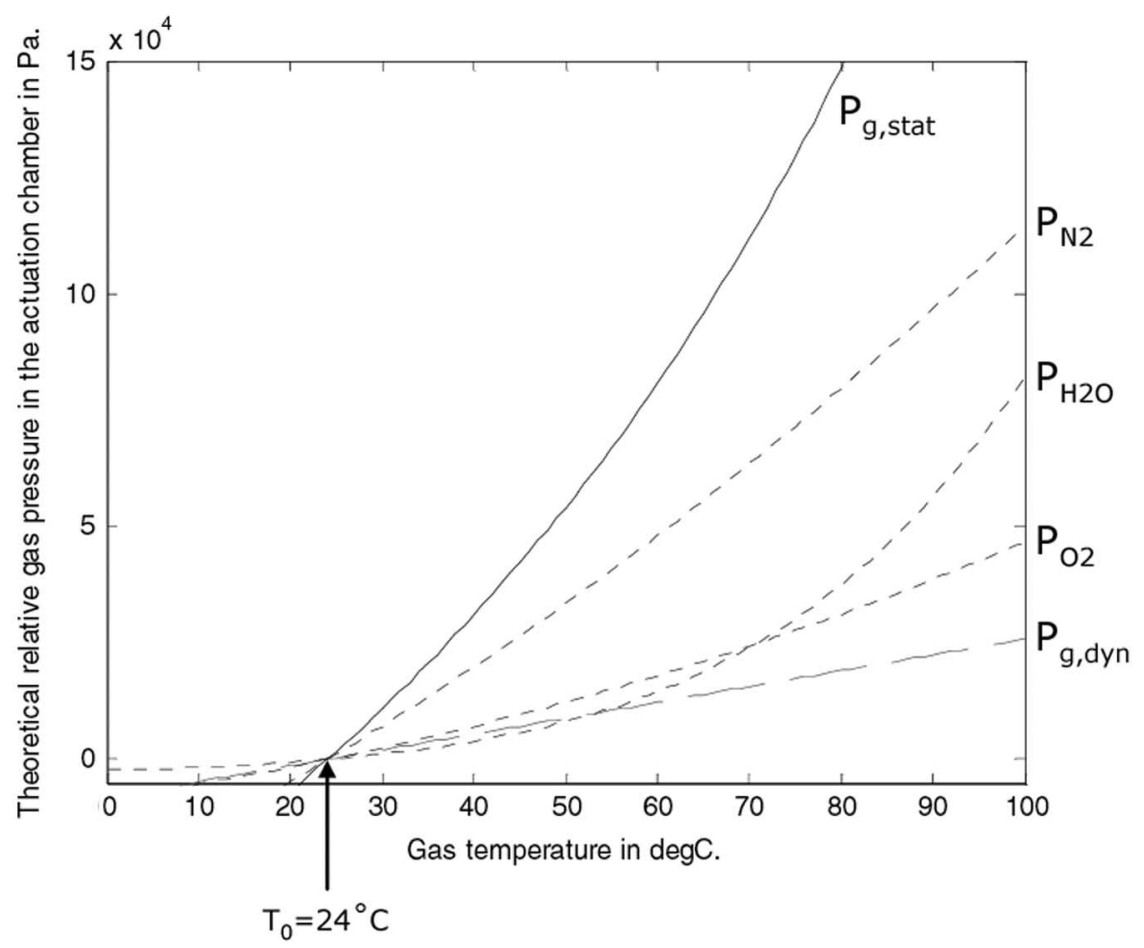

Fig. 3. Plot of the theoretical stationary partial pressure for (blue dotted) $\mathrm{O}_{2}, \mathrm{~N}_{2}$, and vapor (6), (black solid) theoretical overall gas pressure in stationary behavior (7), and (red dashed) dynamic behavior (9) versus the gas temperature $T_{g}$ for a priming temperature $T_{0}=24{ }^{\circ} \mathrm{C}$.
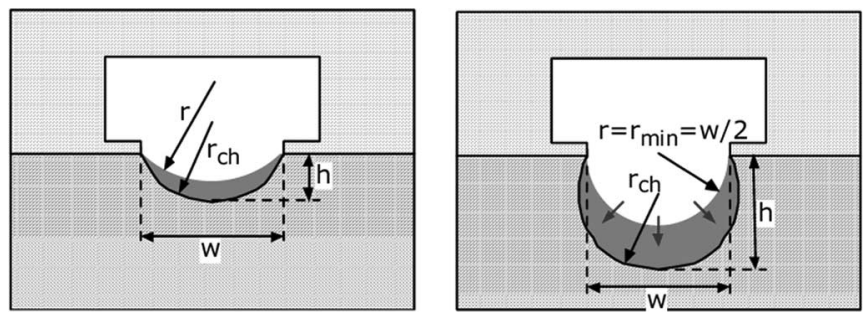

Fig. 4. Schematic cross-sectional view of two valves with a cylindrical channel with radius $r_{\mathrm{ch}}$ and a circular actuation window with diameter $w$. The liquid-air surface radius $r$ depends on the pressure difference over the interface.

The relation between the gas pressure $P_{g}$ and the liquid displacement $\Delta V$ is unambiguously determined by the Young-Laplace equation

$$
P_{g}-P_{l}=\gamma \cdot\left(\frac{1}{r_{1}}+\frac{1}{r_{2}}\right)
$$

for every point of the gas-liquid interface, using the actuation window geometry as boundary condition.

However, for a 3-D model, (10) is difficult to solve analytically when the actuation window is not circular, i.e., the interface shape is not spherical. Therefore, in the following discussion, we will limit ourselves to the analysis of the specific valve geometry of a circular actuation window ${ }^{1}$ with diameter $w$ and a cylindrical liquid channel with radius $r_{\mathrm{ch}}$, as illustrated in Fig. 4.

\footnotetext{
${ }^{1}$ The closing temperature for a circular window is the highest for any window geometry with characteristic dimension $w$, i.e., noncircular window valves will close at lower temperatures
}

For this geometry, (10) simplifies to

$$
P_{g}-P_{l}=\frac{2 \gamma}{r} .
$$

If $w / 2>h$ (Fig. 4, left), the valve closes when $r=r_{\mathrm{ch}}$, i.e., for

$$
P_{g}-P_{l}=\frac{2 \gamma}{r_{\mathrm{ch}}} .
$$

If $w / 2<h$ (Fig. 4 , right), the gas bubble expands suddenly with increasing gas pressure once its radius passes the geometrical minimum

$$
r_{\text {min }}=w / 2
$$

and the closing pressure difference is

$$
P_{g}-P_{l}=\frac{4 \gamma}{w} .
$$

A further theoretical discussion on design parameters and valve failure modes is made in Section VI, where we compare theoretical considerations with experimental results.

For completeness, we will discuss the main assumptions that were made in the aforementioned model. First, we have neglected the solubility of gas in solid. Solubility of vapor and air in permeable materials, such as polydimethylsiloxane (PDMS), will influence the device behavior [9]. The dynamics of gas solubility and gas diffusion in solids are rather slow, however, and their effect is expected to occur as a gradual decrease in gas pressure in the actuation chamber $P_{g}$ in steady state. From this perspective, low-permeable materials should thus be considered for the actuation chamber. 


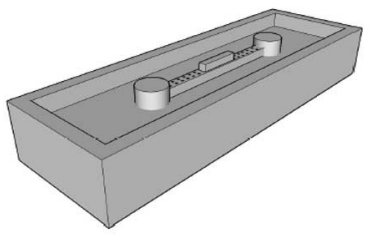

(a)

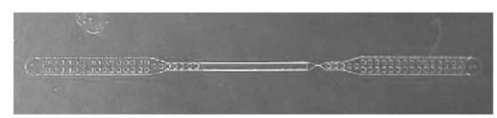

(d)

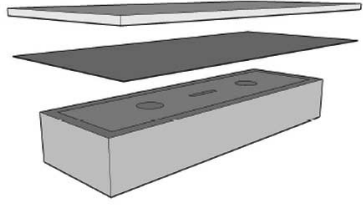

(b)

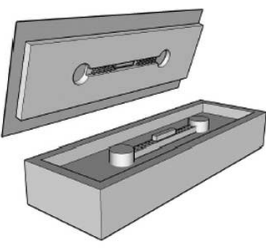

(c)

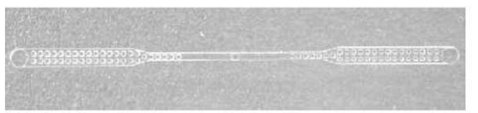

(e)

Fig. 5. (a)-(c) Subsequent schematic of the fabrication of the thin PDMS sheet. (d) Microscopic pictures of PDMS sheets with $100 \times 1000 \mu \mathrm{m}^{2}$ actuation window and necked channel. (e) Microscopic pictures of PDMS sheets with $100 \times 100 \mu \mathrm{m}^{2}$ actuation window without neck. (a) The silicon master is coated with a $\mathrm{CF}_{x}$ polymer. (b) Excess PDMS is squeezed out, and the mold is covered with Scotchpak release liner and pressed with a glass piece. (c) After curing, the PDMS sheet can be easily removed from the mold and from the master.

The assumption of a constant temperature-independent concentration profile $c_{i}$ of the air constituents in water evolves from considering the flux of dissolved air constituents through the liquid $\left(J_{i}\right)$, which can be written as

$$
J_{i}=-D_{i} \Delta c_{i}+L_{i} \Delta T .
$$

$L_{i}$ is a coefficient that depends on the Onsager heat of transport, which describes the thermomigration of the gas constituent through water in the presence of a thermal gradient. Neglecting the thermomigration effect $\left(L_{i}=0\right)$, and considering the stationary state, i.e., $J_{i}=0$, the concentration gradient $\Delta c_{i}$ must be zero as well.

Furthermore, the evaporation enthalpy $\Delta H_{\text {vap }}$ and the surface tension $\gamma$ were assumed constant due to their limited temperature dependence. The evaporation enthalpy decreases slightly $(<10 \%)$ with increasing temperature in the range of interest $0{ }^{\circ} \mathrm{C}<T<100{ }^{\circ} \mathrm{C}$. The surface tension decreases also with temperature, with $d \gamma / d T=0.167 \mathrm{mN} / \mathrm{m}$ for pure water in the same temperature range [10].

Finally, (3) is valid only for systems with constant solvent concentration. Solvents in the water form an entropic barrier for evaporation and thus reduce the saturation vapor pressure $P_{v}$; thus, the change in solvent concentration will change the vapor saturation pressure [11]. For example, the saturation pressure of a completely saturated $\mathrm{NaCl}$ solution in water is only $75 \%$ of that of pure water.

\section{Demonstrator Fabrication}

We fabricated and successfully tested a number of mesoscale microvalve demonstrators. The specific device layout and fabrication method and materials were chosen to allow a quick, uncomplicated, and flexible fabrication and valve interfacing method rather than to manufacture an optimized device in terms of the required actuation temperature, operational robustness, or minimum physical size.

The demonstrator valves are three-layered structures consisting of: 1) a glass substrate; 2) a thin PDMS sheet containing the liquid channel and actuation window; and 3) a thick PDMS block containing fluidic interconnect channels for the valve inlet and outlet and the actuation chamber. The liquid channel in the PDMS sheet contains square pillar structures for mechanical support during fabrication.

The thin PDMS sheet fabrication, as illustrated in Fig. 5, involves the casting of PDMS on a $\mathrm{CF}_{x}$-coated silicon mold in a process that is adapted from Hsu et al. [12]. The silicon master was fabricated using two subsequent deep reactive-ion etching steps and was coated with $\mathrm{CF}_{x}$ polymer using plasma deposition to ease the release of the PDMS sheet after molding. PDMS prepolymer (SYLGARD 184 silicone elastomer kit, Dow Corning) was mixed with the curing agent (10:1 weight ratio) and degassed for $30 \mathrm{~min}$ to remove the air bubbles. The mixture is dispensed on top of the silicon master, and a fluorocarbon polymer-coated polyester (PE) sheet (Scotchpak, 1020 release liner, $3 \mathrm{M}$ ) is carefully rolled over the PDMS, avoiding air bubbles. All excess PDMS is squeezed out by thumb since it is important that there is no material that is left on the top of the silicon mold. The PDMS is finally evened out by pressing a thick piece of glass onto the top surface. Thereafter, the PDMS sheet is cured at $65^{\circ} \mathrm{C}$ for $4 \mathrm{~h}$. After curing, the PDMS adheres reversibly to the PE sheet and is removed from the master along with the PE sheet.

The thick PDMS block is cast in a mold. The mold inserts are removed after the PDMS is cured. A $100-\Omega$ Pt resistance temperature detector (Heraeus M-FK 222) with a size of $0.8 \times 2.2 \times 2.3 \mathrm{~mm}^{3}$ is used simultaneously as heater and temperature sensor and manually placed inside the actuation chamber. Its electrical leads are punched through the PDMS for contacting.

Fig. 6 illustrates the assembly of the valve. First, the PDMS sheet is peeled from the PE sheet and placed on the glass substrate, creating a reversible bond. Thereafter, the thick PDMS block is bonded on top of the PDMS sheet by applying gentle pressure.

The dimensions and materials of the valve were chosen for the fast and uncomplicated fabrication of demonstrator devices and only for ease of evaluation. For use in practical applications, other materials may be more suited, and the device dimensions can be reduced.

\section{EXPERIMENTAL SETUP}

A measurement setup was built, as illustrated in Fig. 7. When the syringe pump is in the OFF state, the inlet and outlet 

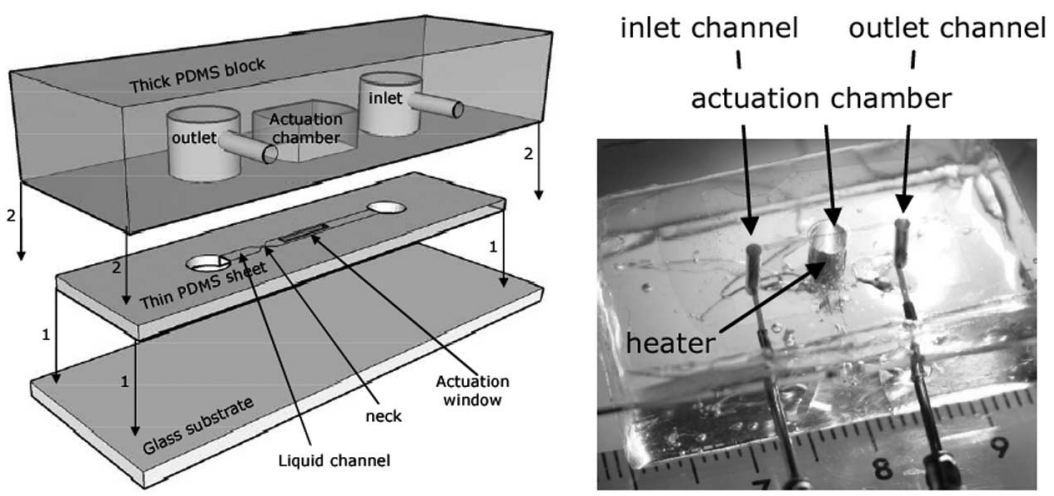

Fig. 6. Left: Exploded schematic of the device assembly with the subsequent bonding steps indicated. Right: Photograph of demonstrator device after priming with red dye.

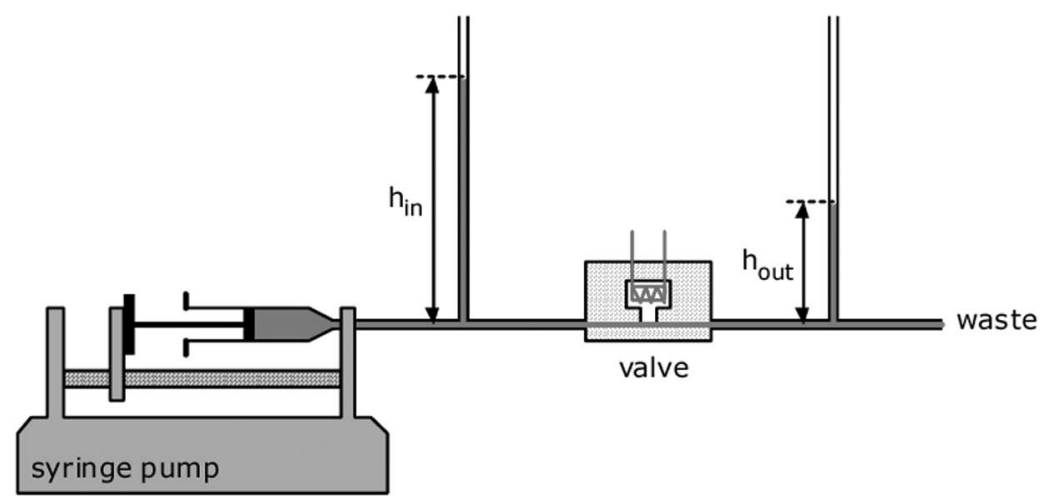

Fig. 7. Schematic of the measurement setup.
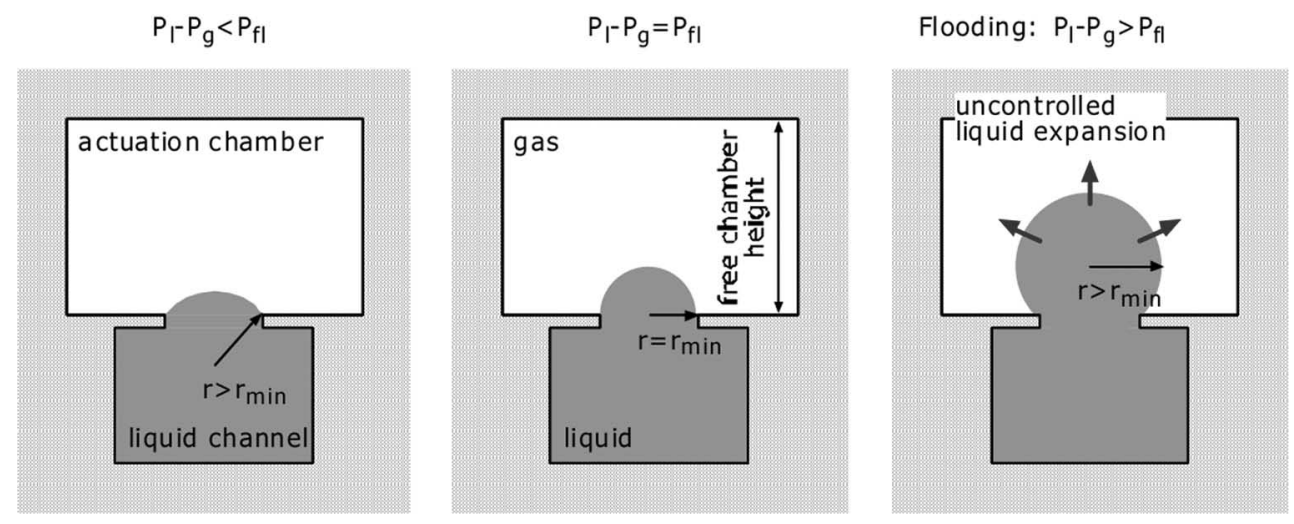

Fig. 8. Left to right: Schematic of the liquid-gas interface shape for increasing $P_{l}-P_{g}$.

\section{FAILURE Mechanisms}

pressures can be applied hydrostatically by setting appropriate values for $h_{\text {in }}$ and $h_{\text {out }}$. Alternatively, a flow can be established through the device with the syringe pump, whereas the inlet and outlet pressures are measured hydrostatically by monitoring the values of $h_{\text {in }}$ and $h_{\text {out }}$. The heater is coupled to a voltage source, and voltage and current were continuously monitored. In the following valve characterization, we used deionized water to which we added red dye for visualization, as well as detergent for easy priming of the structure. Adding surfactant to water will cause its surface tension to drop to a typical approximate value of $\gamma=25 \mathrm{mN} / \mathrm{m}$ (see, e.g., [13]).
This section discusses three identified device failure mechanisms: 1) downstream bubble introduction [7]; 2) flooding [7]; and 3) parasitic offset pressure due to nonequilibrium priming conditions. Downstream bubble introduction refers to when the liquid in the channel cuts off a gas bubble from the actuation chamber and drags it along in the channel. The latter occurs when the liquid pressure drop $P_{\text {in }}-P_{\text {out }}$ over the actuation window region of the valve is larger than the capillary pressure in the liquid channel neck. The neck width must thus be made small enough to withstand the liquid pressure range to be controlled by the valve. Care must also be taken that the neck 


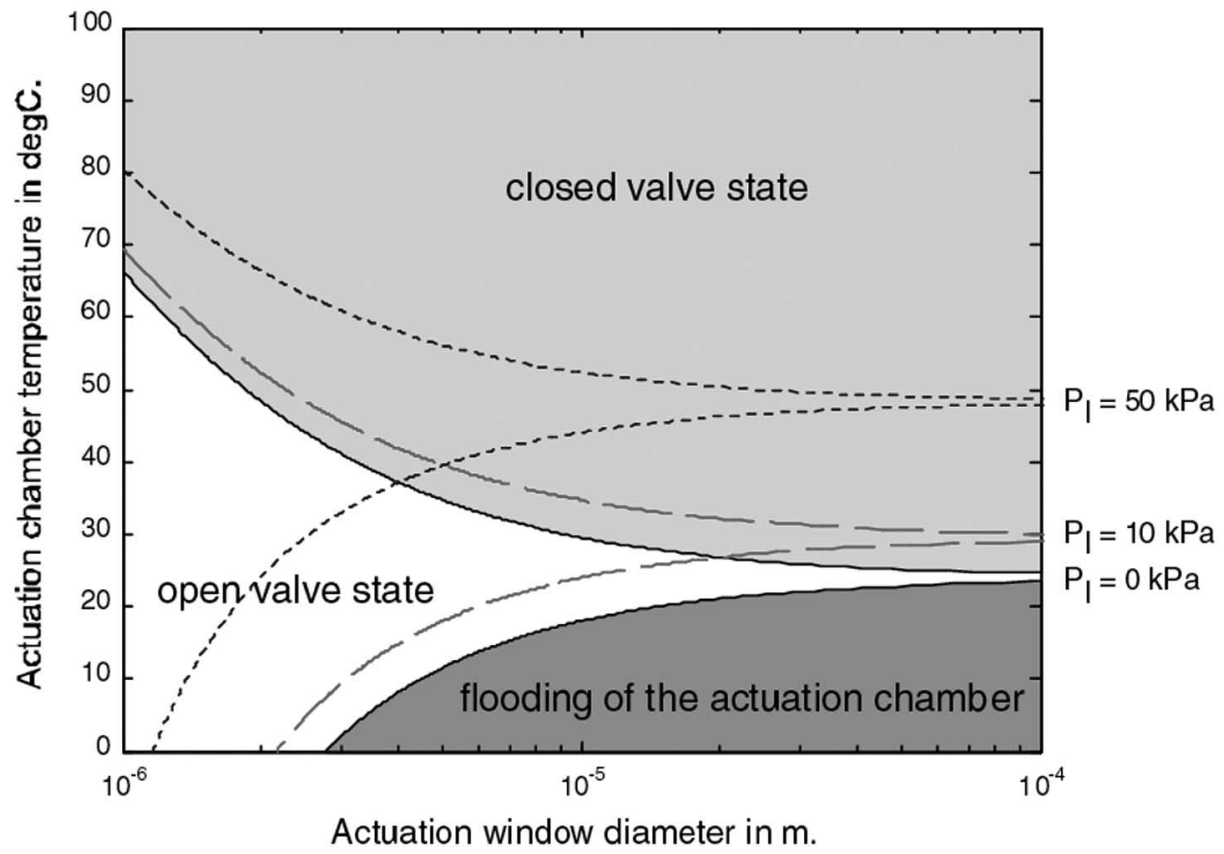

Fig. 9. Theoretical stationary valve closing temperature $T_{\text {close }}$ versus actuation window size at different liquid pressures $P_{l}(0 \mathrm{kPa}$ : black solid line; $10 \mathrm{kPa}$ : red dashed line; $50 \mathrm{kPa}$ : blue dotted line), assuming a circular actuation window with diameter $w$, a cylindrical liquid channel with $w / 2<h$, a priming temperature $T_{0}=24^{\circ} \mathrm{C}$, and a liquid surface tension $\gamma=25 \mathrm{mN} / \mathrm{m}$. The light gray shaded area indicates the closed valve state (at $P_{l}=0 \mathrm{kPa}$ ); the white area indicates the open valve state (at $P_{l}=0 \mathrm{kPa}$ ); and the dark shaded area indicates flooding of the device (at $\left.P_{l}=0 \mathrm{kPa}\right)$.

shape does not lead to an undesired geometric passive valve function during priming [14]. During our experiments, bubble introduction was observed regularly for valves without channel necking, but never for valves with neck.

Flooding, as schematically described in Fig. 8, refers to when the liquid pressure drop $P_{l}-P_{g}$ forces the solid-liquid-gas triple phase line to detach from the actuation window and enter the actuation chamber. For circular actuation window shape and the actuation chamber surface being hydrophobic, flooding occurs when

$$
P_{l}-P_{g}>P_{\mathrm{fl}}
$$

with

$$
\frac{2 \gamma}{w}<P_{\mathrm{fl}}<\frac{4 \gamma}{w} .
$$

Parasitic offset pressure $P_{\text {off }}$ refers to changes in the device behavior that are caused by specific atmospheric conditions at the time of liquid priming. When the atmospheric humidity is below saturation during priming, the air that is trapped in the actuation chamber will spontaneously start adsorbing vapor. The chamber pressure $P_{g}$ will thus increase to a level above the atmospheric pressure with a specific offset, depending on the atmospheric humidity. This effect decreases the required actuation power, which could be positive, but can potentially lead to failure by closing the valve already at atmospheric temperature. It is possible that for small enough valve size, diffusion allows a local thermodynamic equilibrium close to the liquid-gas surface during priming, thus reducing the difference between vapor pressure and saturation vapor pressure during the trapping of the gas in the actuation chamber. The latter has not yet been verified experimentally, however.

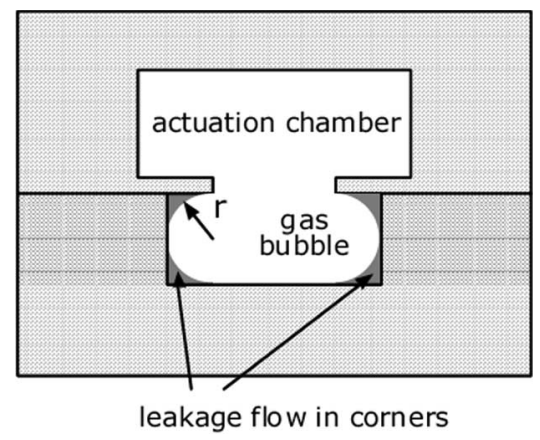

Fig. 10. Schematic of leakage corner flow around the gas bubble in a "closed" valve.
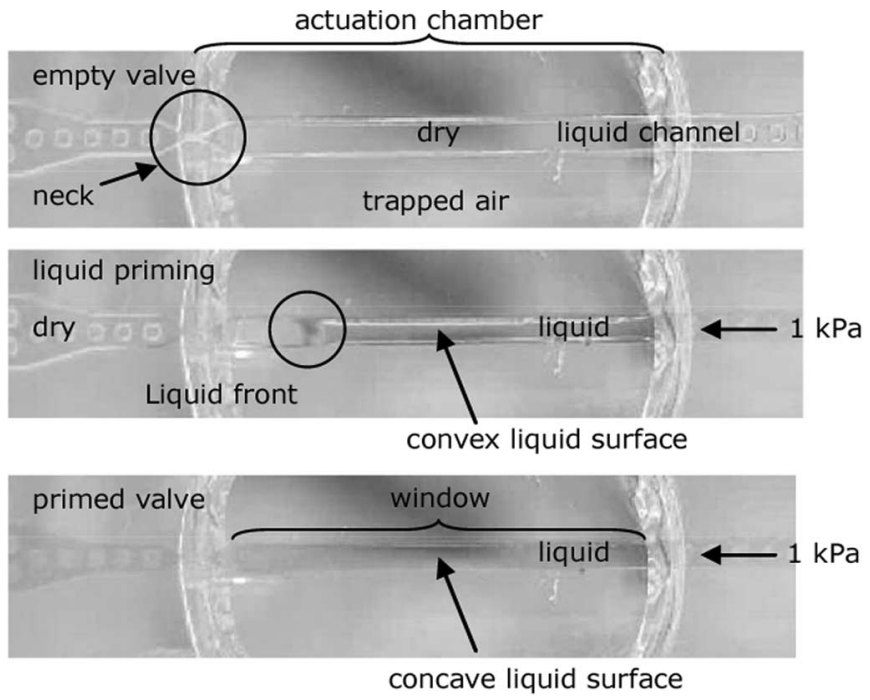

Fig. 11. Subsequent video frames before, during, and after priming of the microvalve. 

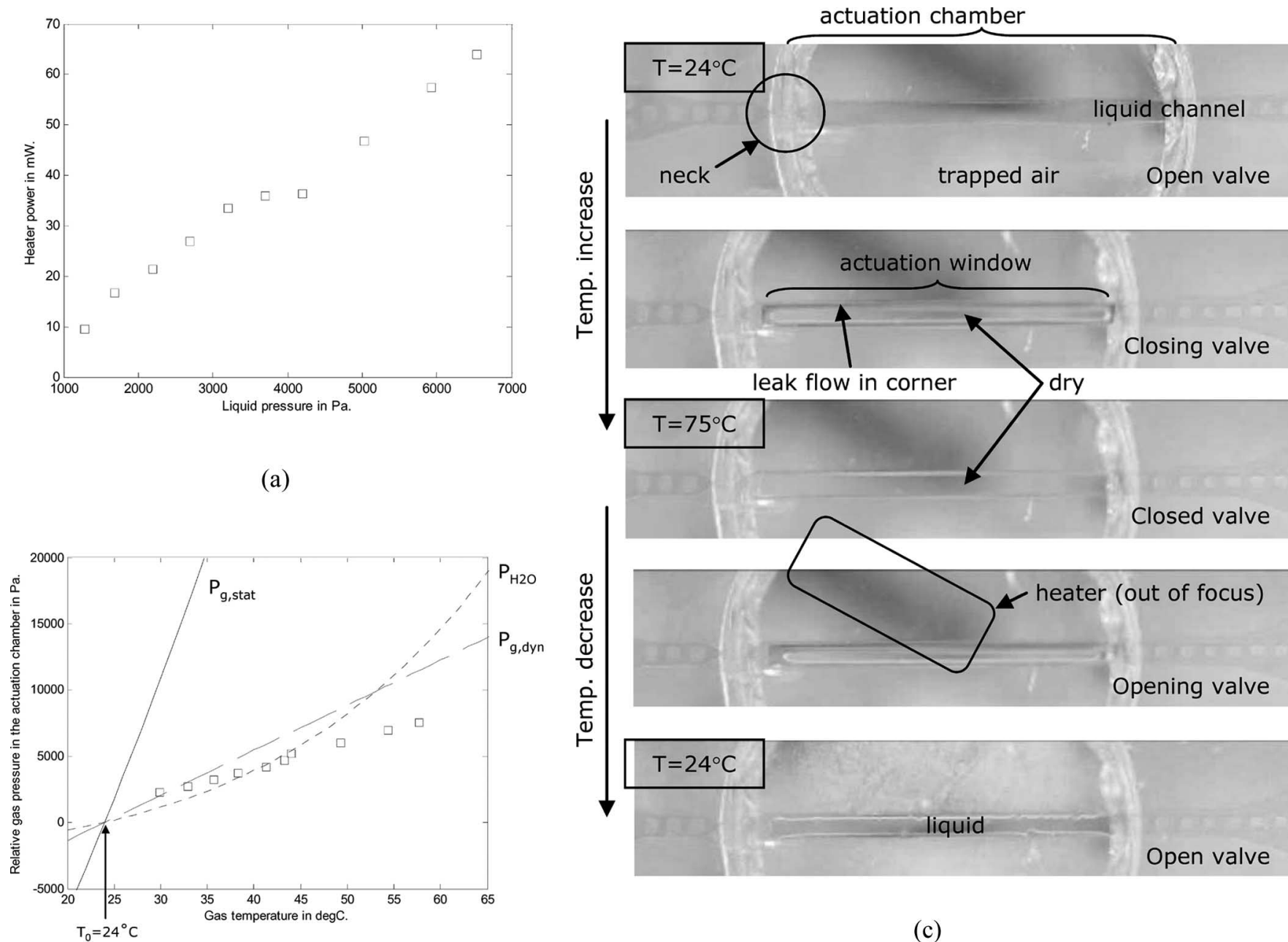

(a)

(c)

(b)

Fig. 12. (a) Applied heating power to close the valve against a set liquid pressure. (b) Correspondingly calculated gas pressure and calculated heater temperature for the same measurement data (squares), with the theoretical overall stationary pressure, dynamic pressures, and vapor saturation pressure (plotted in solid black, dashed red, and dotted blue, respectively). (c) Subsequent video frames (top to bottom) of the opening and closing of a valve with rectangular actuation window.

\section{DESIGN PARAMETERS}

This section discusses the influence of the design parameters on the device performance.

Fig. 9 shows the required closing temperature as a function of the actuation window diameter according to (7) and (14).

Downscaling the actuation window (size $w$ ) increases the required closing temperature $T_{\text {close }}$ (7) but reduces the risk for overflow (16). Increasing the actuation window size leads to decreased robustness, i.e., limiting the temperature and pressure range for which the valve can be in the open state. Fig. 9 indicates the performance that one can expect from the different sized actuator. For example, controlling a pressure-driven flow system with liquid pressure up to $10 \mathrm{kPa}$ and a valve with $10-\mu \mathrm{m}$ actuation window size requires a temperature rise of only $11{ }^{\circ} \mathrm{C}$.

The volume of the actuation chamber does not influence the static system behavior. However, reducing its volume will help in reducing the dynamic system behavior. Care should also be taken that there is a minimum free chamber height $(>w / 2)$ between the actuation window and the roof of the actuation chamber to prevent a protruding liquid front from wetting the inner walls of the actuation chamber, which could cause flooding.

The exact cross-sectional shape of the liquid channel can determine the gas-liquid pressure that is required to close the valve completely. For example, corners in the channel give rise to corner flow around a closing gas bubble (Fig. 10) [7] and will require larger closing pressure.

Note that the valve principle can be used for liquids different than water if the liquid-solid-gas interaction allows pinning the liquid-gas interface in the actuation window (avoiding flooding) in the temperature range of interest.

\section{MeAsurements}

After coupling the valve in the measurement setup, the valve was primed by applying a 1-kPa hydrostatic pressure at the inlet, as shown in Fig. 11.

Under the experimental conditions in our laboratory, we measured typical offset pressure values $P_{\text {off }}=400 \mathrm{~Pa}$ and 
a flooding pressure $P_{\mathrm{fl}}=2 \mathrm{kPa}$ for a valve with a $200-\mu \mathrm{m}$ square actuation window. Note that the unexpectedly large robustness against overflow [(16) predicts $P_{\mathrm{fl}}<500 \mathrm{~Pa}$ ] may be explained by a rather large value of $P_{\text {off }}$ during the particular measurement.

In a second set of measurements on a device with a square actuation window of $w=100 \mu \mathrm{m}$, the power that is required to close the valve against a specific liquid pressure was determined (Fig. 12, left top). For each subsequent measurement, both the inlet and outlet pressures were increased to an equal value, i.e., with zero liquid flow. Thereafter, the power through the heater was gradually increased until it was optically verified that the valve was closed. The measurement results were the pressure values that were obtained at the moment that we could no longer visually observe changes in the shape of the liquid-air interface. It typically took a few to several minutes between the subsequent measurements to reach this point.

Based on these measurements, the actuation pressure that is generated in the actuation chamber can be estimated by adding an estimated phase interface pressure drop $P_{g}-P_{l} \cong$ $(4 \gamma / w)=1 \mathrm{kPa}$ to the applied liquid pressure. The gas pressure is plotted versus the heater temperature in Fig. 12 (left bottom), together with the theoretically expected dynamic gas pressure increase (9) (red dashed), the theoretically expected stationary gas pressure increase (7) (black solid), and the theoretically expected vapor saturation pressure (blue dotted). The theoretical values assume that $V_{0} \gg \Delta V$, with saturated air at $24{ }^{\circ} \mathrm{C}$ room temperature at the time of measurement and at atmospheric pressure as initial conditions.

We believe that the demonstrator valve in this measurement series reaches equilibrium vapor pressure but not equilibrium pressures for the dry air constituents, i.e., it operates somewhere between a pure dynamic and a pure stationary regime. The change in vapor composition during operation can be deduced from the occurrence of a parasitic offset pressure, as aforementioned. That the dry air composition probably remains rather unchanged can be deduced from that the observed pressure increase remains far below the theoretical stationary pressure, as can be seen in Fig. 12(b). The latter is also expected considering that changing the dry gas composition requires the long diffusion path of the air constituents from the environment through the liquid channel to the actuation chamber.

One should also consider that the PDMS material allows for substantial diffusion of gas out of the actuation chamber. This will attribute to the difference between the experimental and theoretical pressures that can be observed in Fig. 12(b).

That the closing temperature during the operation of the particular device depicted in Fig. 12(c) is large as compared to the device measured in Fig. 12(b) is probably due to an improper heat resistor mounting in the former during device assembly.

\section{CONCLUSION}

We introduced a novel type of microfluidic actuator. We provided a theoretical model for qualitative and quantitative analyses of its operational parameters. We successfully built and tested a demonstrator valve with the novel actuator. The absence of moving mechanical parts, the electrical control of the valve, and the limited required actuation temperature make the actuator suitable for control of LSI fluidic networking in biotechnical applications.

\section{REFERENCES}

[1] H.-P. Chou, M. A. Unger, and S. R. Quake, "A microfabricated rotary pump," Biomed. Microdevices, vol. 3, no. 4, pp. 323-330, Dec. 2001.

[2] J. Liu, M. Enzelberger, and S. Quake, "A nanoliter rotary device for polymerase chain reaction," Electrophoresis, vol. 23, no. 10, pp. 15311536, May 2002.

[3] D.-S. Meng, Y. Ju, and C. J. Kim, "A comparative study of electrolysis and boiling for bubble-driven microactuations," in Proc. Transducers, Seoul, Korea, 2005, pp. 1263-1266.

[4] K. Handique, D. T. Burke, C. H. Mastrangelo, and M. A. Burns, "Onchip thermopneumatic pressure for discrete drop pumping," Anal. Chem., vol. 73, pp. 1831-1838, 2001.

[5] S. Böhm, "A closed-loop controlled electrochemically actuated microdosing system," J. Micromech. Microeng., vol. 10, no. 4, pp. 498-504, 2000.

[6] J.-C. Yoo, M.-C. Moon, C. J. Kang, and Y.-S. Kim, "Thermopneumaticactuated PDMS microvalve and micropump integrated with the same fabrication process," in Proc. Tranducers, Seoul, Korea, 2005, pp. 664-667.

[7] J. Melin, W. van der Wijngaart, and G. Stemme, "Behaviour and design considerations for continuous flow closed-open-closed liquid microchannels," Lab Chip, vol. 5, no. 6, pp. 1-6, May 2005.

[8] R. Sander. (1999, Apr. 8). Compilation of Henry's Law Constants for Inorganic and Organic Species of Potential Importance in Environmental Chemistry, Version 3. [Online]. Available: http://www.mpch-mainz. mpg.de/ sander/res/henry.html

[9] K. Hosokawa, "Power-free PDMS microfluidic devices for gold nanoparticle-based DNA analysis," Lab Chip, vol. 4, no. 3, pp. 181-185, Jun. 2004.

[10] M. van Spengen, R. Puers, and I. De Wolf, "A physical model to predict stiction in MEMS," J. Micromech. Microeng., vol. 12, no. 5, pp. 702-713, 2002.

[11] M. J. Blandamer, J. B. F. N. Engberts, P. T. Gleeson, J. Carlos, and R. Reis, "Activity of water in aqueous systems; A frequently neglected property," Chem. Soc. Rev., vol. 34, no. 5, pp. 440-458, 2005.

[12] C.-H. Hsu, C. Chen, and A. Folch, "Microcanals for micropipette access to single cells in microfluidic environments," Lab Chip, vol. 4, no. 5, pp. 420-424, 2004.

[13] B. S. Aksoy, "Hydrophobic forces in free thin films of water in the presence and absence of surfactants," Ph.D. dissertation, Faculty Virginia Polytechnic Inst. State Univ., Blacksburg, 1997.

[14] P. F. Man, C. H. Mastrangelo, M. A. Burns, and D. T. Burke, "Microfabricated capillarity-driven stop valve and sample injector," in Proc. MEMS, Heidelberg, Germany, 1998, pp. 45-50.

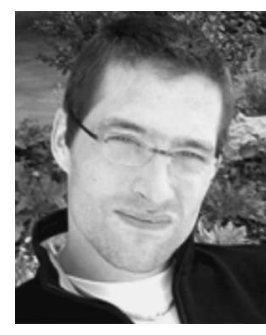

Wouter van der Wijngaart (M'06) received the M.Sc. degree in electrotechnical engineering, the degree of philosophic academy, and the mathematics education degree, all from the Katholieke Universiteit Leuven, Leuven, Belgium, in 1996 and the $\mathrm{Ph} . \mathrm{D}$. degree in microsystem technology from the Royal Institute of Technology (KTH), Stockholm, Sweden, in 2002.

In 2005, he became an Associate Professor at $\mathrm{KTH}$. He is currently leading the micro- and nanofluidics research at the Microsystem Technology Laboratory at KTH. He is the overall Project Manager of the European Framework Six (FP6) project "Q2M - batch integration of high quality materials to microsystems" and leading the microfluidics work of the European FP6 project "SABIO-ultra high sensitive slot waveguide biosensor." $\mathrm{He}$ is also a Cofounder of three companies in the fields of mobile parking payments, microvalving, and microfuel cells.

Prof. van der Wijngaart received, together with Fredric Ankarcrona, the Cap Gemini Innovation Award in the 1999 European Business Plan of the Year Competition. He was also awarded the Swedish Innovation Cup 2001, together with Prof. Andersson. 
Dipankar Chugh received the B.Sc. degree in chemical engineering from the Indian Institute of Technology, India.

He is currently with Microsystem Technology, School of Electrical Engineering, Royal Institute of Technology, Stockholm, Sweden.

Elisabeth Man received the M.Sc. degree in electrical engineering from the Royal Institute of Technology, Stockholm, Sweden, in 1996.

She is currently with the Microsystem Technology, School of Electrical Engineering, Royal Institute of Technology.

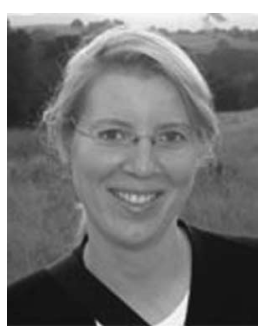

Jessica Melin received the B.Sc. degree from Simon Fraser University, Burnaby, BC, Canada, in 1998, and the Ph.D. degree in microsystem technology from the Royal Institute of Technology, Stockholm, Sweden, in 2004.

She is currently the Director of the Stanford Microfluidics Foundry, Department of Bioengineering, Stanford University, Stanford, CA.

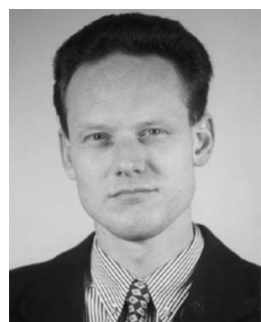

Göran Stemme (M'98-SM'04-F'06) received the M.Sc. degree in electrical engineering in 1981 and the Ph.D. degree in solid state electronics in 1987, both from the Chalmers University of Technology, Gothenburg, Sweden.

In 1981, he joined the Department of Solid State Electronics, Chalmers University of Technology where in 1990, he became an Associate Professor (Docent) heading the silicon sensor research group. In 1991, he was appointed a Professor at The Royal Institute of Technology, Stockholm, Sweden, where he heads the Microsystem Technology group at the Department of Signals, Sensors and Systems. He has published more than 100 research journal and conference papers and has been awarded eight patents. His research is devoted to microsystemtechnology based on micromachining of silicon.

Dr. Stemme was a member of the International Steering Committee of the Conference series IEEE Microelectromechanical Systems (MEMS), between 1995 and 2001, and he was a General Co-chair of that conference in 1998. $\mathrm{He}$ is a member of the Editorial Board of the IEEE/ASME JOURNAL OF MicRoELECTROMECHNICAL SYSTEMS and of the Royal Society of Chemistry journal Lab On A Chip. In 2001, he won, together with two colleagues, the final of the Swedish Innovation Cup. 